\section{Oral Presentation Session 1}

\section{STI Surveillance}

\subsection{A TALE OF TWO HALVES; LOW EXTENDED-SPECTRUM CEPHALOSPORIN AND HIGH AZITHROMYCIN RESISTANCE IN NEISSERIA GONORRHOEAE IN EUROPE, 2015}

${ }^{1}$ Michelle Cole, ${ }^{2}$ Gianfranco Spiteri, ${ }^{3}$ Susanne Jacobsson, ${ }^{1}$ Francesco Tripodo, ${ }^{1}$ Neil Woodford, ${ }^{3}$ Magnus Unemo, ${ }^{2}$ Euro-Gasp Network. 'National Infection Service, London, UK; ${ }^{2}$ European Centre of Disease Prevention And Control, Stockholm, Sweden; ${ }^{3}$ Örebro University Hospital, Örebro, Sweden

\subsection{6/sextrans-2017-053264.1}

Introduction The European Gonococcal Antimicrobial Surveillance Programme aims to monitor rates of antimicrobial resistance in Neisseria gonorrhoeae and provide data to inform the European gonorrhoea treatment guidelines; currently ceftriaxone $500 \mathrm{mg}$ plus azithromycin (Az) $2 \mathrm{~g}$ as dual-therapy. This analysis reports on Euro-GASP resistance patterns in 2015 vs. 2014.

Methods For 2015, antimicrobial susceptibility testing (Etest or agar dilution) was performed on 2134 isolates from 24 European countries and interpreted using EUCAST breakpoints. Patient variables associated with resistance were established using univariate logistic regression to estimate odds ratios (ORs).

Results Cefixime resistance (Cef-R) was $1.7 \%$ in 2015 vs. $2.0 \%$ in 2014 . Ceftriaxone resistance was detected in one isolate in 2015, compared with 5 in 2014. The proportion of isolates showing $\mathrm{Az}$ resistance (Az-R) decreased slightly to 7.1\% (7.9\% in 2014). Five isolates displayed high-level Az-R (MIC $\geq 256 \mathrm{mg} / \mathrm{L}$ ) compared with one in 2014. Ciprofloxacin resistance remained high at $49.4 \%(50.7 \%$ in 2014). Cef-R among MSM and females continued to decline to $0.5 \%$ and $1 \%$, respectively, but increased among heterosexual males $(4.1 \%$ vs. $1.7 \%$ in 2014$)$, which was mainly attributable to two countries with high Cef-R (11\%). MSM and heterosexual males had higher levels of Az-R (both 8.1\%) compared with females (4.9\%), but this difference was not significant. Az-R in females increased to $4.9 \%$ from $2.2 \%$ in 2014. An association between previous gonorrhoea infection and Az-R, first observed in 2014 remained in 2015 (OR 2.1, CI 1.2-3.5, $\mathrm{p}<0.01)$

Conclusion The low cephalosporin resistance likely reflects the effectiveness of the current first-line dual-therapy. However, the Az-R is concerning and threatens the effectiveness of this regimen. Furthermore, the increasing cef- $\mathrm{R}$ in heterosexual males needs close monitoring. Increasing AzR and decreasing cephalosporin resistance is occurring globally. Whether the global use of $\mathrm{Az}$ in mono- or dual-therapy is contributing to this picture remains to be elucidated.

\subsection{PREDICTORS OF PERSISTENT AND RECURRENT GENITAL STI SYMPTOMS AT SENTINEL SURVEILLANCE SITES IN SOUTH AFRICA, JANUARY 2015 - JUNE 2016}

${ }^{1}$ Mathebula Rudzani Climentine, ${ }^{1} \mathrm{~L}$ Kuonza, ${ }^{2} \mathrm{~T}$ Kufa-Chakezha. ${ }^{1}$ South Africa Field Epidemiology Training Program, University of Pretoria, Johannesburg, South African Republic, ${ }^{2}$ HIV and STI Centre, National Institute For Communicable Disease, Johannesburg, South African Republic

\subsection{6/sextrans-2017-053264.2}

Introduction South African guidelines recommend a syndromic approach (SA) to manage sexually transmitted infections (STIs). However, SA lacks specificity and may be particularly problematic for patients with persistent or recurrent genital STI symptoms (PRGS) with inability to adequately distinguish the two. The study objectives were to determine the prevalence and risk factors of PRGS.

Methods We conducted a cross-sectional study at 7 STI sentinel sites in 5 provinces. Demographic, clinical information and clinical specimens were collected. Multivariable logistic regression analysis was used. PRGS was defined as patients who self-reported non-resolution of genital symptoms in the preceding three months or previous treatment for the same genital STI symptoms in the preceding 12 months.

Results Of 1397 eligible patients, 28.9\% reported PRGS. Among those with PRGS, recurrence was more common $90.1 \%$ than persistence $21.8 \%$ with $13.2 \%$ reporting both. The median age of those who reported PRGS was 28 years [IQR: 24-33], with 56.6\% females. Among those with PRGS, $49.3 \%$ had vaginal discharge syndrome (VDS), 33.4\% had male urethral syndrome (MUS) and 17.1\% had genital ulcer syndrome (GUS). The most common pathogens identified among VDS, MUS and GUS patients with PRGS were bacterial vaginosis (60\%), Neisseria gonorrhoea (81.5\%), and herpes simplex virus 2 (57.5\%), respectively. There were no pathogens detected in $19.8 \%$ patients. In multivariable analysis, being HIV-positive (adjusted odds ratio $[\mathrm{aOR}]=1.52$, 95\% CI 1.16-2.00), having VDS $(\mathrm{aOR}=1.66,95 \%$ CI $1.23-2.25)$, being seen at a study site in Gauteng province $(\mathrm{aOR}=0.63$, 95\% CI $0.45-0.89)$ and age between 25-34 years $(\mathrm{aOR}=1.43$, 95\% CI 1.06-1.92) were significantly associated with having PRGS, after adjusting for potential covariates.

Conclusion We found high prevalence of PRGS, associated with HIV infection and VDS among young adults. We therefore recommend better integration of HIV and STI management, increasing HIV testing, and review of SA for VDS to include microbiological testing for pathogens associated with PRGS.

\subsection{ABSTRACT WITHDRAWN}

001.4 HIGH PREVALENCE OF HEPATITIS C VIRUS AMONG HIV NEGATIVE MSM IN AMSTERDAM PREP PROJECT

${ }^{1}$ Roel Achterbergh, 'Elske Hoornenborg, 'Maarten F Schim Van Der Loeff, ' ${ }^{1}$ di Davidovich, ${ }^{1}$ Arjan Hogewoning, ${ }^{2}$ Henry Jc De Vries, ${ }^{1}$ Maria Prins, ${ }^{2}$ Janke Schinkel, ${ }^{3}$ Thijs Jw Van De Laar. 'Public Health Serice Amsterdam, Amsterdam, The Netherlands; ${ }^{2}$ Amsterdam Medical Centre, Amsterdam, The Netherlands; ${ }^{3}$ Sanquin Blood Supply, Amsterdam, The Netherlands

10.1136/sextrans-2017-053264.3 